\title{
Clinical and Laboratory Findings in Dogs Undergoing Adjuvant Chemotherapy with Gemcitabine/Carboplatin Combination for Mammary Neoplasia
}

\author{
Carolina Bistritschan Israel ${ }^{1}$, Tábata Maués ${ }^{2 *}$, Ana Maria Reis Ferreira ${ }^{3}$, and Maria de Lourdes Gonçalves Ferreira ${ }^{3}$ \\ ${ }^{1}$ Luis Cataldi de Souza Veterinary Teaching Clinic, Faculty of Veterinary Medicine, Centro Universitário Serra dos Órgãos, Estrada Wenceslau José de Medeiros, 1045, \\ Prata, Teresópolis, RJ, 25976-345, Brazil \\ ${ }^{2}$ Professor Firmino Mársico Filho Veterinary Teaching Hospital (HUVET), Faculty of Veterinary, Universidade Federal Fluminense, Avenida Almirante Ary Parreiras, \\ 507, Icaraí, Niterói, RJ, 24220-000, Brazil \\ ${ }^{3}$ Department of Pathology and Veterinary Clinic, Faculty of Veterinary, Universidade Federal Fluminense, Avenida Almirante Ary Parreiras, 503, Icaraí, Niterói, RJ, \\ Brazil, 24220-000, Brazil \\ *Corresponding author's Email: tabatamaues@id.uff.br; (D) ORCiD: 0000-0002-8890-608X
}

\begin{abstract}
Adjuvant chemotherapy might be indicated in some canine mammary cancer cases due to metastatic potential. In this regard, studies to determine adverse events following chemotherapy protocols are valuable. The purpose of this prospective clinical trial was to evaluate the safety and tolerability of gemcitabine and carboplatin combination in dogs with malignant mammary tumors. For this prospective clinical trial, 21 female dogs mastectomized due to malignant mammary neoplasia underwent adjuvant chemotherapy with gemcitabine $(3 \mathrm{mg} / \mathrm{kg}$, 60 -minute IV infusion) and carboplatin (10 mg/kg, 20-minute IV infusion) based protocol every 21 days for three cycles. They were monitored periodically for treatment-related adverse events by clinical and laboratory evaluations. A total of 17 $(80.9 \%)$ dogs developed leukopenia, $10(47.6 \%)$ neutropenia, and $15(71.4 \%)$ thrombocytopenia at least once along with the three chemotherapy cycles. All these hematologic toxicities were grade 1,2, or 3. Two (9.5\%) animals had evidence of gastrointestinal toxicity; however, clinical signs were mild to moderate (grades 1 and 2). No dog had life-threatening adverse events (grade 4) or even died (grade 5) of treatment-related complications. The adjuvant chemotherapy protocol with gemcitabine and carboplatin was well-tolerated and safe in female dogs for mammary cancer treatment with self-limiting hematological and gastrointestinal adverse events.
\end{abstract}

Keywords: Adverse event, Canine, Mastectomy, Toxicity, Tumor

\section{INTRODUCTION}

Mammary tumors are the most common neoplasms in female dogs. They have genetic, anatomopathological, and clinical similarities with human breast cancer. The recommended treatment for canine mammary cancer is surgical excision (Cassali et al., 2020; Sorenmo et al., 2020). Adjuvant chemotherapy is indicated in some cases of malignant neoplasms due to metastatic potential (Lavalle et al., 2012) although it might lead to several side effects, mainly myelosuppression and gastrointestinal (GI) disorders (Rodaski et al., 2008). The toxicity occurs since chemotherapeutic agents do not target exclusively neoplastic cells, but high mitotic rate cells, including the regular ones (Rodaski et al., 2008; Gustafson and Bailey, 2020).

Chemotherapy protocols in companion animals may be linked to a good quality of life and extended long-term survival. However, the potential for adverse events that impact the overall quality of life should be taken into consideration (Vail, 2009). This emphasizes the importance of detailed studies on the drug doses and administration frequencies in veterinary cancer patients in order to increase success rates with as few side effects as possible (Medeiros, 2017). Gemcitabine is an antineoplastic drug that requires cell absorption and intracellular phosphorylation to act (Garnett et al., 2016). Doses ranging from 350 to $1,000 \mathrm{mg} / \mathrm{m}^{2}$ (IV) are known to cause the least side effects (Lana et al., 2007; Dominguez et al., 2009) related to hematologic, dermatological, GI, and renal disturbances (Rodaski et al., 2008). Gemcitabine administration has also been found to sensitize the neoplastic cell to a better carboplatin action (Silva et al., 2018). Carboplatin belongs to the second generation of platinum compounds. The drug acts through covalent binding to DNA through displacement reactions resulting in bifunctional lesions and intrastrand crosslinks (Fichtinger-Schepman et al., 1985). Carboplatin has less toxicity than other anticancer agents from the same group (Wagstaff et al., 1989). Side effects observed are nausea and vomiting, nephrotoxicity as well as myelosuppression with neutropenia and thrombocytopenia (Selmic et al., 2014). Lavalle et al. (2012) demonstrated that dogs with malignant mammary tumors treated with carboplatin after surgical excision had a statistically significant longer overall survival when compared with animals submitted to surgical treatment alone. 
An in vitro study showed that gemcitabine and carboplatin decreased cell proliferation, increased apoptosis, and induced cell cycle disruption. Cell cycle interruption and apoptosis were higher when both drugs were administered in sequence (Anjos et al., 2012). The true utility of gemcitabine might act as a potentiator of carboplatin cytotoxicity (Dominguez et al., 2009). The success of combination chemotherapy over single-agent treatment is providing maximal cell kill within the range of tolerable toxicity, accomplishing more interaction between the chemotherapeutic agents and tumor cell population, and slowing the development of cellular drug resistance (Gustafson and Bailey, 2020). Gemcitabine and carboplatin in combination therapy have been evaluated for the treatment of canine osteosarcoma (McMahon et al., 2011) and varied carcinomas (Dominguez et al., 2009). In addition, it is one of the regimens recommended for mammary cancer in dogs (Cassali et al., 2020) although there are no previous studies on toxicity and safety of gemcitabine and carboplatin doublet therapy for canine malignant mammary tumors as far as one can tell from the literature.

In veterinary medicine, there is a lack of studies on the toxicity of chemotherapy regimens. In this context, the aim of this prospective clinical trial was to assess the adverse events, safety, and tolerability of adjuvant chemotherapy with gemcitabine and carboplatin combination for mammary cancer in female dogs through clinical and laboratory evaluations.

\section{MATERIALS AND METHODS}

\section{Ethical approval}

The study was approved by the Ethics Committee on the Use of Animals of Universidade Federal Fluminense with the verdict number 279/12. In addition, written informed consent was obtained from owners to authorize the study.

\section{Patients}

The present study was conducted at the Professor Firmino Mársico Filho Veterinary Teaching Hospital, Universidade Federal Fluminense, Brazil from March 2012 to December 2013.

Dogs were enrolled in this prospective study according to the following criteria: (1) at least one histopathologically confirmed malignant mammary neoplasia; (2) surgical treatment of disease by unilateral or bilateral mastectomy in cases of nodules in one or both mammary chains, respectively; (3) no previous history of cancer; and (4) disease stage I to IV by modified TNM staging system based on WHO classification (Owen, 1980), which means the absence of distant metastasis suggestion by 3-view radiographic examination of the thorax and abdominal ultrasonography.

After the mastectomy, mammary chain resected were fixed in $10 \%$ buffered formalin solution for 48 hours, embedded in paraffin, and cut in $5 \mu \mathrm{m}$ sections. Tumor sections were stained by hematoxylin and eosin, then classified according to Cassali's histopathological criteria (Cassali et al., 2020). Tumor histological type and grade, regional lymph node status, and resection margin status were obtained. These data associated with cancer disease staging and owner willingness to offer an antineoplastic treatment determined whether or not to start adjuvant chemotherapy. In cases of multiple types of mammary tumors in the same dog, the clinical decision-making for adjuvant chemotherapy treatment was based on the most aggressive tumor type and grade.

\section{Chemotherapy administration}

The chemotherapy protocol consisted of three cycles. The interval between cycles was 21 days. Dogs received gemcitabine at a dose of $3 \mathrm{mg} / \mathrm{kg}$ (Rodaski and De Nardi, 2008) IV over 60 minutes. The carboplatin was administered 5 minutes after gemcitabine through the same catheter at a dose of $10 \mathrm{mg} / \mathrm{kg}$ (Rodaski and De Nardi, 2008; Dominguez et al., 2009) IV for 20 minutes. Before, between, and after chemotherapy administrations, $25 \mathrm{ml} / \mathrm{kg} / \mathrm{hour}$ of $0.9 \%$ saline solution was administered as recommended for platinum-derived protocols (Repetti and Daleck, 2007). As chemotherapy-induced toxicity prophylaxis, ondansetron hydrochloride $(0.5 \mathrm{mg} / \mathrm{kg}$ PO every 12 hours $)$ was given for 6 days and starting 3 days before each chemotherapy session for all dogs. In addition, immediately before anticancer agents infusion, ondansetron $(0.5 \mathrm{mg} / \mathrm{kg} \mathrm{IV})$, ranitidine hydrochloride $(1.0 \mathrm{mg} / \mathrm{kg} \mathrm{SC})$ and promethazine $(1.0 \mathrm{mg} / \mathrm{kg} \mathrm{IV})$ were administered (Viana, 2007).

\section{Adverse events evaluation}

Adverse events were graded according to the Veterinary Cooperative Oncology Group - Common Terminology Criteria for Adverse Events following investigational therapy in dogs and cats (LeBlanc et al., 2021) based upon clinical and laboratory evaluations (E). About 20 days after mastectomy and before the first cycle of adjuvant chemotherapy, baseline clinical and laboratory evaluations (E0) were performed. Table 1 shows the schedule of chemotherapy cycles as well as clinical and laboratory evaluations. Dogs were evaluated on days 0, 15, 20, 36, 41, 57 and 62, identified as E0, E1, E2, E3, E4, E5 and E6, respectively, for this 63-day study. The female dogs were subjected to blood count tests from 
E0 to E6 and serum biochemistry and urinalysis with urine protein-creatinine ratio exams in E0, E2, E4, and E6. These findings were accounted for as laboratory changes. Clinical evaluation and complete blood counts (including manual white blood cell differential) were also performed on E1, E2, and E5. Clinical evaluation from E0 to E6 included physical examination, abdominal palpation, cardiopulmonary auscultation, oral, and ocular mucosa examination, as well as monitoring of weight, temperature, and hydration status. Besides, clinical history and anamnesis were carried out with the dog's owner with regard to appetite, vomiting, diarrhea, lethargy, and other non-ordinary events related to antineoplastic drugs side effects.

Table 1. Schedule of clinical and laboratory evaluations (E0 to E6) and the three cycles of gemcitabine and carboplatin combination chemotherapy performed on female dogs with mammary cancer

\begin{tabular}{|c|c|c|c|c|c|c|c|}
\hline \multirow{3}{*}{ Days } & \multicolumn{5}{|c|}{ Evaluation } & \multirow{2}{*}{\multicolumn{2}{|c|}{ Chemotherapy }} \\
\hline & \multirow[b]{2}{*}{ Period } & \multirow[b]{2}{*}{ Clinical } & \multicolumn{3}{|c|}{ Laboratory } & & \\
\hline & & & Blood count & $\begin{array}{c}\text { Serum } \\
\text { biochemistry }\end{array}$ & Urinalysis & Session & Cycle \\
\hline 0 & E0 & $\mathrm{x}$ & $\mathrm{x}$ & $\mathrm{x}$ & $\mathrm{x}$ & & \\
\hline 1 & & & & & & $\mathrm{x}$ & First \\
\hline 15 & E1 & $\mathrm{x}$ & $\mathrm{x}$ & & & & \\
\hline 20 & $\mathrm{E} 2$ & $\mathrm{x}$ & $\mathrm{x}$ & $\mathrm{x}$ & $\mathrm{x}$ & & \\
\hline 22 & & & & & & $\mathrm{x}$ & Second \\
\hline 36 & E3 & $\mathrm{x}$ & $\mathrm{x}$ & & & & \\
\hline 41 & E4 & $\mathrm{x}$ & $\mathrm{x}$ & $\mathrm{x}$ & $\mathrm{x}$ & & \\
\hline 43 & & & & & & $\mathrm{x}$ & Third \\
\hline 57 & E5 & $\mathrm{x}$ & $\mathrm{x}$ & & & & \\
\hline 62 & E6 & $\mathrm{x}$ & $\mathrm{x}$ & $\mathrm{x}$ & $\mathrm{x}$ & & \\
\hline
\end{tabular}

E0: Baseline evaluation, E1: First evaluation, E2: Second evaluation, E3: third evaluation, E4: Fourth evaluation, E5: Fifth evaluation, E6: Sixth evaluation, $\mathrm{x}$ : Marks the evaluated parameter.

\section{Adverse events managing}

Toxicity management varied according to tumor type and grade from chemotherapy cycle delay to symptomatic or prophylactic therapy prescription.

\section{Statistical analysis}

In the clinical trial, one-way analysis of variance (Dunn, 1964; Scheffé, 1999) and the paired Student's t-test considered GI toxicosis (emesis, diarrhea, hyporexia, and weight loss) and other clinical toxicity (such as lethargy, fever, hypothermia). The laboratory findings were hematological (complete blood count, global leukometry, neutrophil count, platelet count, red blood cells, haemoglobin, and hematocrit) and metabolic toxicoses (increase in creatinine, urea, alanine aminotransferase [ALT] and alkaline phosphatase [ALP], decrease in serum albumin, and increase in urine protein-creatinine ratio). The abnormal parameters taken were classified into five degrees (LeBlanc et al., 2021).

\section{RESULTS}

\section{Patients' characteristics}

A total of 98 female dogs met the inclusion criteria. From these, 77 were excluded from the current study for the following reasons: (1) not having chemotherapy indication, since they had stage I disease and low aggressive tumors; (2) their owners declining the adjuvant chemotherapy recommendation; or (3) were dogs undergoing a different chemotherapy protocol. Finally, a total of 21 female dogs of varying ages and breeds, including mixed breed dogs, with mammary cancer met inclusion criteria and were enrolled for this prospective clinical trial. Seven (33.3\%) dogs were spayed and $14(66.7 \%)$ dogs were not. The mean age was 10.4 years (range 7-14 years). There were eight (38.09\%) Poodles Toy, four (19.04\%) mixed breed dogs, two (9.52\%) Daschunds, one (4.76\%) Bichon Frise, one (4.76\%) Border Collie, one (4.76\%) Bull Terrier, one (4.76\%) Rottweiler, one (4.76\%) English Cocker Spaniel, one (4.76\%) Brazilian Terrier and one (4.76\%) Pinscher. The mean weight was $11.5 \mathrm{~kg}$ (ranged from 3.1 to $37.5 \mathrm{~kg}$ ) wherein 16 dogs were above $15.0 \mathrm{~kg}$. The time interval between surgery and initiation of chemotherapy was approximately 21 days (range $16-$ 44 days). No significant correlation between toxicity and age, breed, or even weight was found.

\section{Staging, tumor type, and grade}

With regards to disease staging, $47.6 \%(n=10 / 21), 28.6 \%(n=6 / 21), 19.1 \%(n=4 / 21)$ and $4.7 \%(n=1 / 21)$ of the animals were stage I, II, III and IV, respectively. The most common mammary tumor type was carcinoma in a mixed 
tumor grade II $(66.6 \%, \mathrm{n}=14 / 21)$, followed by tubular carcinoma grade II $(19.0 \%, \mathrm{n}=4 / 21)$, papillar carcinoma grade II, solid carcinoma grade III, and carcinosarcoma grade II $(4.8 \%, \mathrm{n}=1 / 21$ each). No significant correlation between toxicity and cancer stage, tumor type, or grade was found. Ten animals received adjuvant chemotherapy due to tumor size over $3 \mathrm{~cm}$ (six dogs stage II plus four dogs stage III) and one because of positive lymph node metastasis (stage IV). Among these animals, two dogs stage III had more aggressive tumor types such as carcinosarcoma grade II and solid carcinoma grade III. All female dogs with stage I cancer had mammary tumor grade II and underwent chemotherapy by owners' voluntary choice. The clients were aware that no scientific evidence of adjuvant chemotherapy benefits for grade II malignant mammary tumors is available, but they were concerned about metastatic disease potential and therefore opted for adjuvant treatment after surgery.

\section{Laboratory findings}

The main hematological changes during all three adjuvant chemotherapy cycles were leukopenia (43 events in 126 blood count exams), neutropenia (24 events in 126 exams), and thrombocytopenia (38 events in 126 exams). About $80.9 \%(\mathrm{n}=17 / 21)$ of the female dogs had leukopenia at least once from E1 to E6 (43 events in 17 dogs). A higher

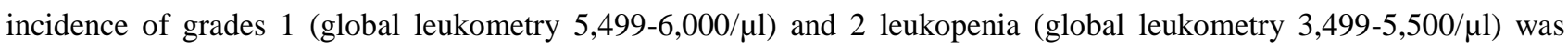

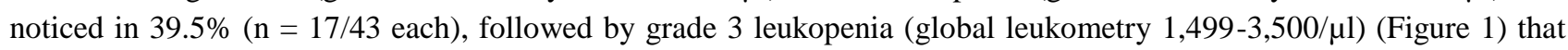
summed up $21.0 \%(n=9 / 43)$ of events. The most severe episodes of leukopenia happened in E1 (blood count 14 days after the first chemotherapy session) and E4 (blood count before the third session). However, only leukopenia in E1 was statistically significant ( $\mathrm{p}<0.05$ ) when compared to leukopenia in E2, E3, E4, E5, and E6.

With regards to neutropenia, $47.6 \%(\mathrm{n}=10 / 21)$ of animals had it at least once from E1 to E6. To sum up all 24 episodes of neutropenia ( 24 events in $10 \mathrm{dogs}), 66.7 \%(\mathrm{n}=16 / 24)$ were grade 1 (Absolute Neutrophil Count [ANC] $1,500-3,000 / \mu 1), 20.8 \%(\mathrm{n}=5 / 24)$ were grade $2($ ANC $1,000-1,499 / \mu 1)$ and $12.5 \%(\mathrm{n}=3 / 24)$ were grade $3($ ANC 500999/ $\mu 1$, Figure 2). Two animals (a $19.7 \mathrm{~kg}$ Mixed Breed dog and a $25.0 \mathrm{~kg}$ Border Collie) had grade 3 neutropenia and one of them (Border Collie) showed afebrile grade 3 neutropenia again later (Neutrophil count $=925 / \mu 1$ ). Neutropenia had a higher frequency and was statistically significant $(\mathrm{p}<0.05)$ on blood count after the first chemotherapy cycle $($ E1). No animal had febrile neutropenia.

Around $38.1 \%$ of female dogs $(n=8 / 21)$ had a delayed or second nadir about 19 days after chemotherapy administration, totalizing 12 neutropenia events in E2, E4, and E6. About 71.4\% ( $\mathrm{n}=15 / 21)$ of the female dogs had thrombocytopenia at least once from E1 to E6 (38 events in 15 dogs). Thrombocytopenia was more frequently detected in the blood count after the second (E3) and third (E5) chemotherapy sessions (Figure 3). It was mild / grade 1 (platelet

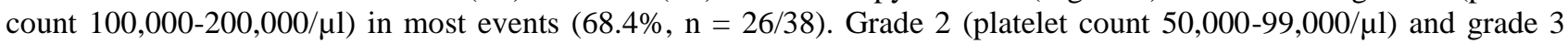

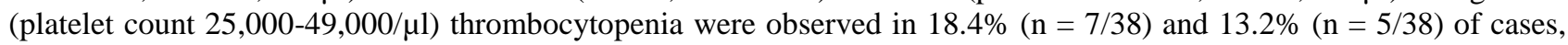
respectively. The decrease in platelet count was highly significant $(\mathrm{p}<0.0001)$ in E3, considerably significant ( $<<$ $0.001)$ in E1 and E5, and significant $(\mathrm{p}<0.05)$ in E2, E4, and E6. The most severe thrombocytopenia cases occurred in E3 and E5 blood counts (both platelet count $=40,000 / \mu 1$ ), performed 14 days after the second and the third chemotherapy sessions, respectively.

No animal had anemia during this clinical trial. Finally, descriptive analyzes of the different events (leukopenia, neutropenia, and thrombocytopenia), related to toxicity degree and chemotherapy cycle, showed a higher hematological adverse event in E1, E3, and E5 control blood counts with E1 being the most severe. It is noteworthy that one 12-yearold Poodle breed dog $(4.7 \%, \mathrm{n}=1 / 21)$ did not present myelosuppression at any time from E0 to E6. No dog developed elevations in hepatic or renal values during treatment. Nonetheless, one animal already had a pre-existing grade 4 elevation in ALT (1,440U/l) and grade 1 in ALP (246U/l) at the beginning of the study and before hepatopathy treatment. No other female dog had abnormalities noted on pretreatment clinical and laboratory evaluations (E0).

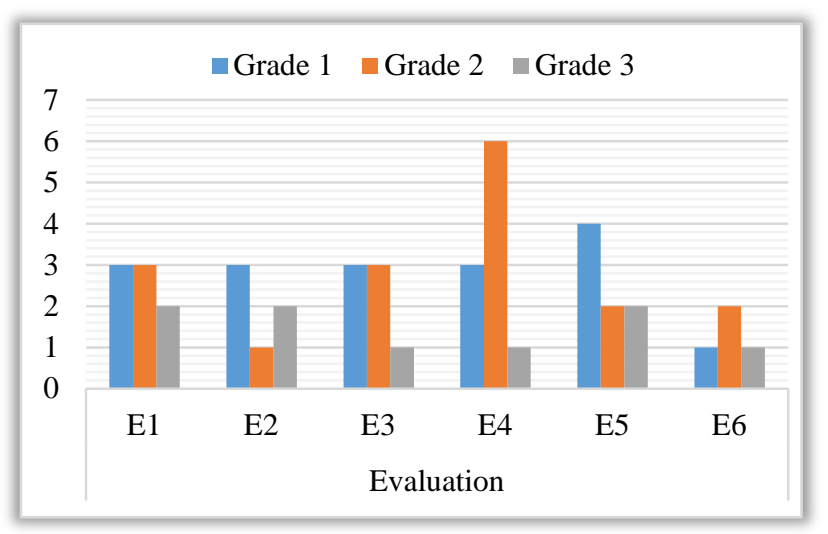

Figure 1. Number of leukopenia events $(n=43)$ distributed in grades from E1 to E6 in 21 female dogs who underwent adjuvant chemotherapy with gemcitabine and carboplatin combination. E1: 14 days (nadir) after first chemotherapy cycle; E2: 2 days before the second chemotherapy cycle; E3: 14 days (nadir) after second chemotherapy cycle; E4: 2 days before the third chemotherapy cycle; E5 and E6: 14 (nadir) and 19 days after third chemotherapy cycle, respectively. 


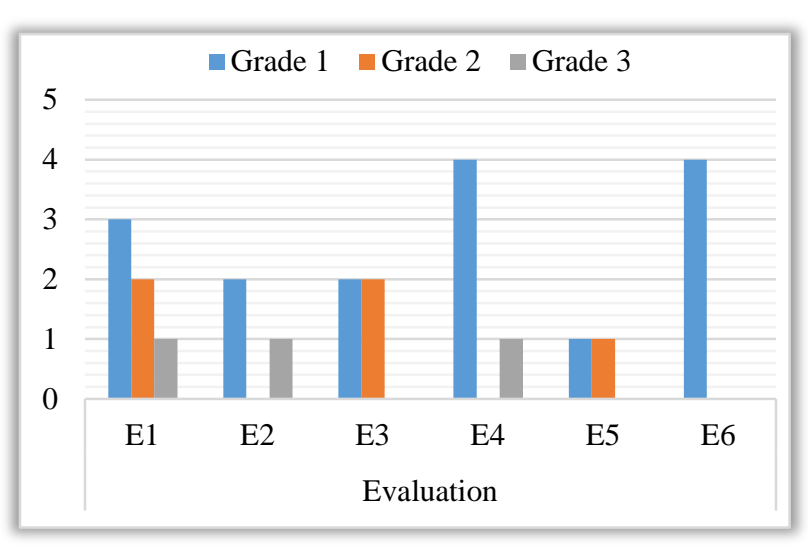

Figure 2. Number of neutropenia events $(n=24)$ distributed in grades from E1 to E6 in 21 female dogs who underwent adjuvant chemotherapy with gemcitabine and carboplatin chemotherapy combination. E1: 14 days (nadir) after first chemotherapy cycle; E2: 2 days before the second chemotherapy cycle; E3: 14 days (nadir) after second chemotherapy cycle; E4: 2 days before the third chemotherapy cycle; E5 and E6: 14 (nadir) and 19 days after third chemotherapy cycle, respectively.

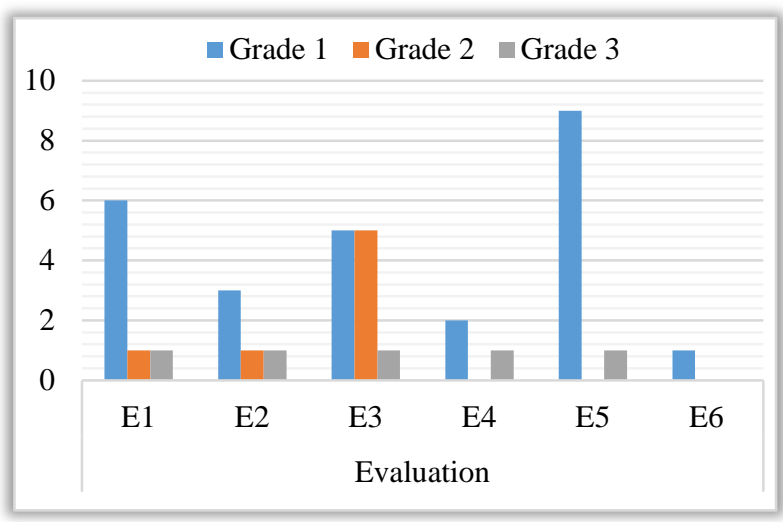

Figure 3. Number of thrombocytopenia events $(n=38)$ distributed in grades from E1 to E6 in 21 female dogs underwent adjuvant chemotherapy with gemcitabine and carboplatin chemotherapy combination. E1: 14 days (nadir) after first chemotherapy cycle; E2: 2 days before the second chemotherapy cycle; E3: 14 days (nadir) after second chemotherapy cycle; E4: 2 days before the third chemotherapy cycle; E5 and E6: 14 (nadir) and 19 days after third chemotherapy cycle, respectively.

\section{Clinical findings}

Clinical signs of GI toxicity were based on episodes of emesis, diarrhea, hyporexia, and weight loss. Only two dogs $(9.5 \%, \mathrm{n}=2 / 21)$ had vomiting and diarrhea both a few days after the first chemotherapy session. One animal, a nine-year-old Bull Terrier, was classified with GI toxicity grade 1, and the other, a seven-year-old Border Collie, grade 2. The constitutional clinical sign of lethargy was reported by owners in three female dogs $(14.3 \%, \mathrm{n}=3 / 21)$ and occurred only after the first chemotherapy cycle, classified as grade 1. Regarding other clinical adverse events, no animal had another GI sign or even other constitutional clinical (fever, hypothermia and weight loss), allergic, cardiac, dermatologic, ear and labyrinth, hemorrhagic, musculoskeletal/soft tissue, neurological, ocular, respiratory nor genitourinary disorders.

\section{Adverse events managing}

Neutropenia, thrombocytopenia, and GI signs lead to adverse events management protocols, which varied according to toxicity type and grade. Neutropenia grades 2 and 3, as well as grade 1 when ANC was under 2,500/ $\mu$, led to treatment delay of 2 to 7 days considering the severity and individual recovery time. Therefore, around $11.2 \%(\mathrm{n}=$ $7 / 63)$ of chemotherapy cycles were postponed due to neutropenia events in E2 $(n=2 / 7)$ and E4 $(n=5 / 7)$ in 5 dogs. The two animals that showed neutropenia under cutoff value in E2, presented neutopenia again in E4. Hemogram was performed every 48 or 72 hours for neutropenic dogs and the chemotherapy session delayed was retaken when ANC

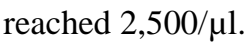

Prophylactic broad-spectrum antibiotic (enrofloxacin $5 \mathrm{mg} / \mathrm{kg}$ PO every 12 hours for 7 days) was prescribed for two dogs with afebrile grade 3 neutropenia with ANC under 750/ $\mu 1$ (both in the nadir of first chemotherapy cycle, one in E1, and other in E2). One of those dogs presented afebrile grade 3 neutropenia once again in E4 (nadir of second chemotherapy cycle) nevertheless antibiotic was not prescribed since ANC was $925 / \mu 1$, which is over $750 / \mu 1$ (Bisson et al., 2020).

Thrombocytopenia grades 3 and 2 when platelets were under 75,000/ $\mu 1$ led to chemotherapy administration delay in 2 to 7 days considering the severity and individual recovery time. Thus, $4.8 \%(\mathrm{n}=3 / 63)$ chemotherapy cycles were postponed due to thrombocytopenia events in $\mathrm{E} 2(\mathrm{n}=2 / 3)$ and $\mathrm{E} 4(\mathrm{n}=1 / 3)$ given that one dog in E2 also had concurrent grade 3 neutropenia $(\mathrm{ANC}=640 / \mu \mathrm{l})$ contributing to session suspension. Hemogram was performed every 48 or 72 hours for thrombocytopenic dogs and the delayed chemotherapy session was retaken when platelets accomplished the cutoff value of $75,000 / \mu 1$.

It is should be noted that neutropenia and thrombocytopenia under cutoff values occasioned chemotherapy delay only in E2 and E4 since those were the evaluations before chemotherapy sessions. Concerning GI adverse events (vomiting plus diarrhea) presented by two animals, diet adjustments, ondansetron (1.0 mg/kg PO every 12 hours), and omeprazole (1.0 mg/kg PO every 24 hours) were prescribed. No antineoplastic drug dosage has been reduced even for animals that previously showed adverse events. No dog had a life-threatening adverse event (grade 4) or even died (grade 5) of chemotherapy treatment-related complications in this clinical trial. No animal was hospitalized due to 
chemotherapy-related adverse events.

\section{Clinical outcomes}

Regarding treatment response, no dog had local recurrence nor metastasis (by 3 -view radiographic examination of the thorax and abdominal ultrasonography 3 months after surgery) during the 63-day study period. After chemotherapy treatment, owners were recommended to bring their dogs for follow-up every three months to evaluate disease recurrence and metastasis. Nevertheless, 19 (90.5\%) dogs lost follow-up along the long-term period of 60 months. Therefore, only two (9.5\%) patients were enrolled in the overall survival time (OST) analysis (from surgical treatment to death). The first was a thirteen-year-old Daschund with carcinoma in a mixed tumor grade 2 and stage I. The latter was an eight-year-old Poodle with carcinoma in a mixed tumor grade 2 and stage III. The OST was 1,125 and 1,797 days, respectively. The cause of both deaths was unknown (information through pet owners' phone contact).

\section{DISCUSSION}

The efficacy of systemic therapy for mammary gland tumors has not been confirmed according to the highest evidencebased standards, even though chemotherapy is routinely recommended for dogs with large tumors (tumor size $>3 \mathrm{~cm}$ diameter), lymph node metastasis, and aggressive tumor histology after surgery (Sorenmo et al., 2020) since these features are related to recurrence / distant metastasis and shorter survival time (Rasotto et al., 2017).

Although Coffee et al. (2020) found carboplatin-induced neutropenia and thrombocytopenia especially in dogs under $15.0 \mathrm{~kg}$, the findings of the current study indicated no correlation between myelosuppression and body weight. However, it must be considered that the authors studied a canine population much larger than the current study (101 versus 21) which leads to higher statistical reliability. Interestingly, the dog that had the worst neutropenic events (two episodes of grade 2 neutropenia in E3 and E6 plus two episodes of grade 3 neutropenia in E2 and E4) was a seven-yearold $25 \mathrm{~kg}$ Border Collie. In addition, the same canine had 3 thrombocytopenic events (one grade 1 episode in E5 and two grade 2 events in E2 and E3) and was one out of the two dogs that had vomiting (grade 2) and diarrhea (grade 1) after the first chemotherapy session. Border Collie breed dogs may have polymorphisms (Alves et al., 2011) and even nt230 (del4) ABCB1 gene mutation (Dekel et al., 2017; Marelli et al., 2020), interfering with glycoprotein $\mathrm{P}$ substrates metabolism. Several chemotherapeutic agents have already been identified as substrates of the glycoprotein P (Mealey, 2004; Linardi and Natalini, 2006; Mealey et al., 2017), but gemcitabine and carboplatin were not so far. Thus, no PCR test for $A B C B 1$ mutation was requested for the Border Collie dog that showed exacerbated toxicity compared to other animals studied.

Bone marrow suppression is a frequently encountered chemotherapy toxicity. The bone marrow cells divide rapidly, so it is a prime target since the activity of most anticancer agents is greatest in tissues with a high growth rate. The clinical result of myelosuppression is varying degrees of peripheral blood cytopenias (MacDonald, 2009). Indeed leukopenia, neutropenia, and thrombocytopenia were the most common hematological findings along 126 blood count exams (from E1 to E6) in 21 canines during gemcitabine and carboplatin doublet treatment. However, these blood adverse events were considered acceptable since most were grade 1 and no grade 4 or 5 were found. As with other authors (Dominguez et al., 2009; McMahon et al., 2011), the hematological toxicosis observed in dogs was minimal to moderate. The most frequent side effects in humans were hematological, mainly thrombocytopenia and neutropenia (Maisano et al., 2011). In the current study, leukopenia was also considered statistically significant in dogs in addition to thrombocytopenia and neutropenia. However, it should be considered that only neutropenia and thrombocytopenia are considered for clinical judgment and decision making.

In humans with lung cancer undergoing gemcitabine and carboplatin chemotherapy, a quarter of patients developed myelosuppression grades 3 and 4, as another quarter showed no sign of myelosuppression (Gréen et al., 2016). In the current study, about one-third of the animals $(28.6 \%, n=6 / 21)$ had grade 3 leukopenia at some point of the treatment while one female $\operatorname{dog}(4.7 \%, \mathrm{n}=1 / 21)$ did not present myelosuppression at any time. The results of the current study are similar to another research in dogs (Dominguez et al., 2009) that found grades 3 and 4 neutropenia in $32 \%$ of canines $(n=12 / 37)$ and to a pilot study in cats (Martinez-Ruzafa et al., 2009) in which two animals $(14.3 \%, n=2 / 14)$ developed grade 3 or 4 neutropenia. However, grades 3 and 4 neutropenia were substantially more frequent (65\%) with the same antineoplastic drugs in humans (Usami et al., 2010). Regarding this discrepancy in toxicity, the doses employed for humans are different from dogs and cats, and so is the incidence of adverse events.

Neutropenia is usually the most serious and dose-limiting cytopenia associated with chemotherapeutic drug administration. Animals with neutropenia that are afebrile and asymptomatic should have chemotherapy delayed for a few days to one week based on the severity (MacDonald, 2009), as it was done in the current study in $11.2 \%$ of chemotherapy cycles $(n=7 / 63)$. Even though neutropenia was detected in $47.6 \%(n=10 / 21)$ of dogs in the current study, febrile neutropenia was observed in none of the cases as expected since this event is more common with other 
anticancer agents use, such as vincristine, doxorubicin (Britton et al., 2014) and lomustine (Cunha et al., 2017). Pyrexia accompanying neutropenia is a more clinical concern than if a patient is afebrile and clinically well, as it indicates the probable presence of infection (Bisson et al., 2018). Since all neutropenia reported in this clinical trial were afebrile and dogs did not show alterations at clinical screening or the owner's questionnaire, antimicrobial prophylaxis was not prescribed for animals with grades 1 and 2 neutropenia. Two animals had grade 3 neutropenia, both clinically well, but due to ANC under 750/ $\mu 1$, antibiotic was prescribed according to Bisson et al. (2020) and Fournier et al. (2017) recommendation for ANC cutoff for antimicrobial prophylaxis. The enrofloxacin was chosen for antimicrobial prophylaxis due to other authors' guidance (Thamm and Vail, 2007; Vail, 2009; Boudreaux, 2014; Gustafson and Bailey, 2020). One of those two dogs showed afebrile grade 3 neutropenia (ANC $=925 / \mu 1$ ) again, but antibiotic was not prescribed this time since ANC was over 750/ $\mu$ l (Bisson et al., 2018; Bisson et al., 2020) and the animal did not have risk factors, such as hematological malignancies, concomitant disease, or weight less than $14 \mathrm{~kg}$ - was a $25 \mathrm{~kg}$ Border Collie (Bisson et al., 2018).

Chemotherapy cycles were delayed in $23.8 \%(n=5 / 21)$ of animals, while McMahon et al. (2011) deferred treatment in $12 \%(n=6 / 50)$ and Dominguez et al. $(2009)$ in $18.9 \%(n=7 / 37)$ of dogs mainly due to neutropenia severity. On the other hand, in a study with cats (Martinez-Ruzafa et al., 2009) receiving gemcitabine (2mg/kg IV) on days 1 and 8 and carboplatin (10mg/kg IV) on day 1, the findings indicated that 6 out of 14 cats $(42.9 \%)$ experienced treatment delays. If instead of 2,500/ $\mu$ l we had opted for a lower ANC cutoff value, such as $1,500 / \mu 1$, as recommended by other authors (MacDonald, 2009), we probably would have reported less delayed sessions.

Dose reductions are deleterious to the optimum delivery of chemotherapy (Gustafson and Bailey, 2020). For that reason, no antineoplastic drugs dosage has been reduced for animals that previously showed adverse events in the current trial inasmuch as no grade 4 toxicity has occurred as recommended by MacDonald (2009) and different from Dominguez et al. (2009) who decided for gemcitabine and carboplatin dose reduction of $25 \%$ as a result of grade 3 neutropenia in one dog. McMahon et al. (2011) administrated the combination of carboplatin and gemcitabine in 50 dogs every 21 days. They reduced carboplatin dosage 15 times in 6 dogs (12\%) during treatment cycles 2 and 3 due to grade 3 and 4 neutropenia, grade 3 thrombocytopenia, grade 2 lethargy, and grade 2 anorexia. Guidelines for dose adjustments of anticancer agents are not standardized, and the administrations are done empirically (10\% to $25 \%$ dose reduction) whenever severe or unacceptable hematologic or GI toxicosis has happened (Gustafson and Bailey, 2020).

The nadir varies with individual drugs, however, it commonly occurs 5-10 days after chemotherapy administration (MacDonald, 2009). Around 38.1\% of female dogs $(n=8 / 21)$ had a delayed or a second nadir about 19 days $(\mathrm{E} 2$, E4, and E6) after the chemotherapy session probably due to carboplatin use (Chun et al., 2007). Thrombocytopenia cutoff value of 75,000/ $\mu$ l followed MacDonald (2009) recommendation. Given the fact that anemia is a rare treatment-related event (MacDonald, 2009), indeed it was expected no anemia report in the current study. Considering that our study lasted 63 days, even if chemotherapeutic agents affected red cells development in bone marrow, we would not have enough time to evaluate since the canine erythrocyte lifespan is about 110 days (Lindena et al., 1986).

A study in dogs receiving gemcitabine $2 \mathrm{mg} / \mathrm{kg}$ by slow intravenous infusion, followed by carboplatin $10 \mathrm{mg} / \mathrm{kg}$ on day 1 , gemcitabine alone on day 8 , and then repeating the cycle on day 21 , reported minimal to moderate hematological toxicity (Dominguez et al., 2009). Another research on dogs with osteosarcoma using gemcitabine $2 \mathrm{mg} / \mathrm{kg}$ followed by carboplatin $300 \mathrm{mg} / \mathrm{m}^{2}$ both by intravenous infusion and repeating the cycle on day 22 concluded that the hematological toxicity of this protocol was acceptable (McMahon et al., 2011). In the present study, hematological adverse events were also considered mild to moderate. Besides, gemcitabine was infused first followed by carboplatin to avoid antagonistic effects. Further, this infusion order is considered more effective since provides synergism between chemotherapeutic agents (Wang et al., 2010).

Even though there are reports of renal function changes related to these drugs (Calvert et al., 1989; Barabas et al., 2008), no renal toxicity degree was observed. It is known that platinum derivatives tend to predispose to renal toxicity. However, when compared to cisplatin, carboplatin has been shown to be significantly less toxic (Dominguez et al., 2009). Moreover, one dog already had increased ALT (grade 4) and ALP (grade 1) activities in E0 (before the first chemotherapy session). Nevertheless, due to hepatopathy treatment (Silimarin $20 \mathrm{mg} / \mathrm{kg}$ every 12 hours PO), those parameters were normalized along with the clinical trial period even with chemotherapy maintenance. The animal presented grade 2 ALT and grade 1 ALP when started the first chemotherapy cycle. Given the dog did not have marked enzyme activities increasing along with the treatment (E1 to E6), hepatotoxicity was not considered to be an important adverse effect of gemcitabine and carboplatin combined protocol. In this context, no degree of treatment-related hepatic or renal toxicity was observed in the current study. Liver toxicity due to gemcitabine has not been observed in humans either (Toschi et al., 2005; Saif, 2010).

In 20 human patients treated with gemcitabine and carboplatin, nausea and vomiting were not commonly observed (Usami et al., 2010) similar to the current study in which only two female dogs $(9.5 \%, \mathrm{n}=2 / 21$ ) had vomiting and diarrhea after the first chemotherapy session and no longer in the subsequent cycles even with the maintenance of antineoplastic agents dosages for following sessions. Martinez-Ruzafa et al. (2009) and Dominguez et al. (2009) studied 
tolerability of gemcitabine ( $2 \mathrm{mg} / \mathrm{kg} \mathrm{IV})$ on days 1 and 8 and carboplatin $(10 \mathrm{mg} / \mathrm{kg}$ IV) on day 1 in cats and dogs, respectively, with carcinomas from different origins. The former found GI toxicity in 4 out of 14 cats (28.6\%) while the latter observed mild to moderate severity and self-limiting GI signs in 27 out of 37 dogs (73.0\%). Although both studies reported higher rates of GI adverse events, compared to the findings of the current study, they administrated gemcitabine twice totalizing $4 \mathrm{mg} / \mathrm{kg}$ for cycle whereas $3 \mathrm{mg} / \mathrm{kg}$ was used once for each chemotherapy cycle in the present research. Using carboplatin and gemcitabine combination every 21 days, McMahon et al. (2011) observed 17 episodes of grade 1 or 2 GI toxicity in $26 \%$ of $\operatorname{dogs}(n=13 / 50)$, including anorexia, nausea, diarrhea, and vomiting. The GI toxicity signs management with diet adjustments, antiemetic (ondansetron), and proton pump inhibitor (omeprazole) was performed according to Gustafson and Bailey (2020) recommendations.

Given the prophylactic medication, the vomiting low rate might be due to ondansetron prescription. It means that the incidence and degree of GI toxicosis may be underestimated in the current study. Nevertheless, Dominguez et al. (2009) also used concurrent metoclopramide as prophylactic antiemetic medication in 22 of 37 dogs and found GI toxicosis in 27 animals $(73 \%, \mathrm{n}=27 / 37)$, which is considerably higher than the results of the present study. This could be related to gemcitabine only once administered in the present protocol or even to ondansetron superiority over metoclopramide as an antiemetic for dogs receiving chemotherapy. Metoclopramide had no effect on either vomiting or nausea for dogs treated with cisplatin while 5-HT3 receptor antagonist ondansetron demonstrates greater antiemetic and anti-nausea efficacy over metoclopramide and maropitant (Kenward et al., 2017).

Less frequent adverse events have been reported in humans by some authors, such as acute myocardial infarction, skin rash, thrombosis, or pulmonary embolism (Fidias et al., 2008; Lee et al., 2009), pulmonary toxicity (Baig et al., 2019), neurotoxicity (Joerger et al., 2002; McWhinney et al., 2009), skin fibrosis (Hashimoto et al., 2011), and skin necrosis (Holstein et al., 2010), even though these effects were not observed in the current study.

Most veterinary patients enjoy a good quality of life while on chemotherapy treatment (MacDonald, 2009) as seen in the present study. Therefore, anticipating and managing adverse events is essential to allow chemotherapeutic administration modifications and improve the quality of life of dogs during treatment (Gustafson and Bailey, 2020).

Chemotherapy may fail due to unacceptable toxicity (Gustafson and Bailey, 2020). The knowledge of gemcitabine and carboplatin doublet therapy main side effects in canine cancer patients, especially in a homogeneous group, including same tumor (mammary neoplasm) and treatment objective (adjuvant therapy following mastectomy), is essential on veterinary oncologist practicing. Understanding drug activity and chemotherapy toxicity, can help the physician in treatment decision making and ensuring dog owners are appropriately educated as to the type and likelihood of adverse events and planning for appropriate preventive and therapeutic protocols to manage them (Vail, 2009; Gustafson and Bailey, 2020).

The lack of commitment of the owners with their animals' follow-up led to insufficient information collection about disease-free intervals and overall survival time. Since we obtained the OST data of only two female canines (OST $=1,125$ and 1,797 days) and no further information, it is not possible to evaluate the efficacy of gemcitabine and carboplatin doublet chemotherapy protocol in reducing disease recurrence and metastasis or increasing survival time.

The current prospective clinical trial had some limitations, including the small number of female dogs, evaluations lasting until the third chemotherapy cycle without further monitoring of most dogs, administration of only one schedule and dosage, and GI toxicity probably underestimated due to prophylactic ondansetron therapy. Therefore, further longterm follow-up studies aiming at survival time, therapeutic gain, maximum tolerated dose, biologically effective dose, and dosing schedule to make these drugs combination use more effective and less toxic as possible are required.

Even though novel drugs are a great promise for cancer treatment in animals, optimizing the use of currently already available agents might bring new benefits (Thamm and Gustafson, 2020). In this regard, both gemcitabine and carboplatin are medications known for decades, but there is still a lack of information about these chemotherapeutic agents association for use in dogs. The current study represented the first assessment of toxicity and safety of gemcitabine and carboplatin given in combination for mammary cancer adjuvant treatment in female dogs.

\section{CONCLUSION}

The adjuvant chemotherapy protocol with gemcitabine and carboplatin combination was well-tolerated and safe in female dogs with mammary cancer. Most hematological and GI adverse events were considered mild to moderate, but all self-limiting.

\section{ECLARATIONS}

\section{Authors' contribution}

All authors have made substantial contributions to all parts of the study. CBI and MLGF designed the project. 
CBI and TM performed animals' selection, surgery, chemotherapy, clinical evaluations, sampling, analysis, and interpretation of data and literature review. AMRF carried out the histopathological analysis. CBI executed data collection. CBI and TM prepared the draft manuscript. TM and MLGF critically reviewed and edited writing. All authors read and approved the final manuscript.

\section{Acknowledgments}

The authors thank all dog owners who authorized the use of the data of their animals. This work was supported by the Coordenação de Aperfeiçoamento de Pessoal de Nível Superior (CAPES) of the Brazilian Ministry of Education.

\section{Competing interests}

The authors declare that they have no competing interests.

\section{REFERENCES}

Alves L, Hulsmeyer V, Jaggy A, Fischer A, Leeb T, and Drogemullee M (2011). Polymorphisms in the ABCB1 Gene in Phenobarbital Responsive and Resistant Idiopathic Epileptic Border Collies. Journal of Veterinary Internal Medicine, 25(3): 484-489. DOI: https://www.doi.org/10.1111/j.1939-1676.2011.0718.x

Anjos CPG, Vasconcelos AC, Crosara PFTB, Anjos GC, Beckere CG, and Guimarães RES (2012). Poptosis in eosinophilic nasal polyps treated in vitro with mitomycin C. Brazilian Journal of Otorhinolaryngology, 78(3): 32-37. Available at: http://www.bjorl.org.br/en-apoptosis-ineosinophilic-nasal-polyps-articulo-S180886941530241X

Baig J, Shokouh-Amiri M, Chan J, Chowdhery R, Danthurthy S, and Venepalli NK (2019). The spectrum of pulmonary toxicity in pancreatic cancer patients receiving gemcitabine combination chemotherapy. Case Reports in Oncology, 12(2): 506-512. Available at: https://www.karger.com/Article/FullText/500242

Barabas K, Milner R, Lurie D, and Adin C (2008). Cisplatin: A review of toxicities and therapeutic applications. Veterinary and Comparative Oncology, 6: 1-18. DOI: https://www.doi.org/10.1111/j.1476-5829.2007.00142.x

Bisson JL, Argyle DJ, and Argyle SA (2018). Antibiotic prophylaxis in veterinary cancer chemotherapy: A review and recommendations. Veterinary and Comparative Oncology, 16(3): 301-310. DOI: https://www.doi.org/10.1111/vco.12406

Bisson JL, Fournier Q, Johnston E, Handel I, and Bavcar S (2020). Evaluation of a 0.75 × 109/L (=750 /uL) absolute neutrophil count cut-off for antimicrobial prophylaxis in canine cancer chemotherapy patients. Veterinary and Comparative Oncology, 18(3): 258-268. DOI: https://www.doi.org/10.1111/vco.12544

Boudreaux B (2014). Antimicrobial use in the veterinary cancer patient. Veterinary Clinics of North America: Small Animal Practice, 44(5): 883-891. Available at: https://www.sciencedirect.com/science/article/abs/pii/S0195561614000825?via\%3Dihub

Britton BM, Kelleher ME, Gregor TP, and Sorenmo KU (2014). Evaluation of factors associated with prolonged hospital stay and outcome of febrile neutropenic patients receiving chemotherapy: 70 cases (1997-2010). Veterinary and Comparative Oncology, 12(4): 266-276. DOI: https://www.doi.org/10.1111/vco.12001

Calvert AH, Newell DR, Gumbrell LA, O'Reilly S, Burnell M, Boxall FE, Siddik ZH, Judson IR, Gore ME, and Wiltshawet E (1989). Carboplatin dosage: prospective evaluation of a simple formula based on renal function. Journal of Clinical Oncology, 7(11): 1748-1756. Available at: https://ascopubs.org/doi/abs/10.1200/JCO.1989.7.11.1748

Cassali GD, Jark PC, Gamba C, Damasceno KA, EstrelaLima A, De Nardi AB, Ferreira E, Horta RS, Firmo BF, Sueiro FAR et al. (2020). Consensus Regarding the Diagnosis, Prognosis and Treatment of Canine and Feline Mammary Tumors - 2019. Brazilian Journal of Veterinary Pathology, 13(3): 555-574. Available at: https://bjvp.org.br/banner/2020/11/v-13-n-3-1/

Chun R, Garret L and Vail D (2007). Cancer chemotherapy. In: Withrow SJ, Vail DM (Editors), Withrow\& MacEwen's Small Animal Clinical Oncology. 4th Ed. Saunders Elsevier, St. Louis, Missouri, USA, pp. 163-192.

Coffee C, Roush JK, and Higginbotham ML (2020). Carboplatin-induced myelosuppression as related to body weight in dogs. Veterinary and Comparative Oncology, 18(4): 804-810. DOI: https://www.doi.org/10.1111/vco.12622

Cunha SCS, Silva FBF, Corgozinho KB, Silva KVGC, and Ferreira AMR (2017). Adverse Effects of Chemotherapy in Dogs. World's Veterinary Journal, 7(3): 74-82. Available at: http://wvj.science-line.com/vol-7-no-3-sep-2017.html

Dekel Y, Machluf Y, Stoler A, Aderet A, Baumel D, Kellerman E, Plotsky Y, Partouche ON, Elhalal G, Ben-Shlomo I et al. (2017). Frequency of canine nt230(del4) MDR1 mutation in prone pure breeds, their crosses and mongrels in Israel - insights from a worldwide comparative perspective. BMC Veterinary Research, 13: 333-341. Available at: https://bmcvetres.biomedcentral.com/articles/10.1186/s12917-017-1251-9

Dominguez PA, Dervisis NG, Cadile CD, Sarbu L, and Kitchell BE (2009). Combined gemcitabine and carboplatin therapy for carcinomas in dogs. Journal of Veterinary Internal Medicine, 23: 130-137. DOI: https://www.doi.org/10.1111/j.1939-1676.2008.0248.x

Dunn OJ (1964). Multiple comparisons using rank sums. Technometrics, 6(3): 241-252. Available at: https://amstat.tandfonline.com/doi/abs/10.1080/00401706.1964.10490181

Fichtinger-Schepman AM, van der Veer JL, den Hartog JH, Lohman PHM, and Reedijk J (1985). Adducts of the antitumor drug cisdiamminedichloroplatinum (II) with DNA: formation, identification, and quantitation. Biochemistry, 24(3): 707-713. Available at: https://pubs.acs.org/doi/pdf/10.1021/bi00324a025

Fidias PM, Dakhil SR and Lyss AP (2008). Phase III study of immediate compared with delayed docetaxel after front-line therapy eith gemcitabine plus carboplatin in advanced nonsmall-cell lung cancer. Journal of Clinical Oncology, 27(4): 591-598. Available at: https://ascopubs.org/doi/pdfdirect/10.1200/jco.2008.17.1405

Fournier Q, Serra JC, Handel I, and Lawrence J (2017). Impact of pretreatment neutrophil count on chemotherapy administration and toxicity in dogs with lymphoma treated with CHOP chemotherapy. Journal of Veterinary Internal Medicine, 32: 384-393. DOI: https://www.doi.org/10.1111/jvim.14895

Garnett CL, Guerrero TA, and Rodriguez Jr CO (2016). Fixed dose-rate administration of gemcitabine in cancer-bearing cats: A pilot study. The Canadian Veterinary Journal, 57(11): 1149-1155. Available at: http://europepmc.org/article/med/27807377

Gréen H, Hasmats J, Kupershmidt I, Edsgard D, Petris L, Lewensohn R, Blackhall F, Vikingsson S, Besse B, Lindgren A et al. (2016). Using WholeExome Sequencing to Identify Genetic Markers for Carboplatin and Gemcitabine-Induced Toxicities. Clinical Cancer Research, $22(2)$ : $366-373$. 
Available at: https://clincancerres.aacrjournals.org/content/clincanres/22/2/366.full.pdf

Gustafson DL, and Bailey DB (2020). Cancer Chemotherapy. In: Vail DM, Thamm DH, Liptak JM (Editors), Withrow \& MacEwen's Small Animal Clinical Oncology. 6th Ed. Saunders Elsevier, St. Louis, Missouri, USA, pp. 182-208.

Hashimoto A, Yamamoto N, Hata Y, Takahashi M, and Shimokawa R (2011). Dermal fibrosis related to combination chemotherapy with carboplatin and gemcitabine. European Journal of Dermatology, 21(6): 999-1001. Available at: https://www.jle.com/fr/revues/ejd/edocs/dermal fibrosis related to combination chemotherapy with carboplatin and gemcitabine 291038/article.phtml

Holstein A, Batge R, and Egberts EH (2010). Gemcitabine induced digital ischaemia and necrosis. European Journal of Cancer Care, 19(3): 408-409. DOI: https://www.doi.org/10.1111/j.1365-2354.2008.01057.x

Joerger M, Gunz A, Speich R, and Pestalozzi BC (2002). Gemcitabine-related pulmonary toxicity. Swiss Medical Weekly, 132(1-2): 17-20. Available at: https://pubmed.ncbi.nlm.nih.gov/11901447/

Kenward H, Elliott J, Lee T, and Pelligand L (2017). Anti-nausea effects and pharmacokinetics of ondansetron, maropitant and metoclopramide in a low-dose cisplatin model of nausea and vomiting in the dog: a blinded crossover study. BMC Veterinary Research, 13(244): 1-12. Available at: https://bmcvetres.biomedcentral.com/articles/10.1186/s12917-017-1156-7

Lana SE, Rutteman GR, and Withrow SJ (2007). Tumors of the mammary gland. In: Withrow SJ, Vail DM (Editors), Withrow\& MacEwen's Small Animal Clinical Oncology. 4th Ed. Saunders Elsevier, St. Louis, Missouri, USA, pp. 619-638.

Lavalle GE, De Campos CB, Bertagnolli AC, and Cassali GD (2012). Canine malignant mammary gland neoplasms with advanced clinical staging treated with carboplatin and cyclooxygenase inhibitors. In Vivo, 26(3): 375-379. Available at: https://pubmed.ncbi.nlm.nih.gov/22523289/

Leblanc AK, Atherton M, Bentley RT, Boudreau CE, Burton JH, Curran KM, Dow S, Giuffrida MA, Kellihan HB, Mason NJ et al. (2021). Veterinary Cooperative Oncology Group - Common Terminology Criteria for Adverse Events (VCOG-CTCAE v2) following investigational therapy in dogs and cats. Veterinary and Comparative Oncology, pp. 1-42. DOI: https://www.doi.org/10.1111/vco.12677

Lee SM, Rudd R, and Woll PJ (2009). Randomized double-blind placebo-controlled trial of thalidomide in combination with gemcitabine and carboplatin in advanced non-small lung cancer. Journal of Clinical Oncology, 27(31): 5248-5254. Available at: https://ascopubs.org/doi/10.1200/JCO.2009.21.9733

Linardi RL, and Natalini CC (2006). Multi-drug resistance (MDR1) gene and P-glycoprotein influence on pharmacokinetic and pharmacodymanic of therapeutic drugs. Ciência Rural, 36: 336-341. Available at: https://www.scielo.br/scielo.php?script=sci arttext\&pid=S0103$\underline{84782006000100056}$

Lindena J, Wittenberg H, Diederichs F, and Trautschold I (1986). The Decline of Catalytic Enzyme Activity Concentration of In Vivo Ageing Erythrocytes of the Man, the Dog and the Rat. Journal of clinical chemistry and clinical biochemistry, 24(1): 49-59. Available at: https://edoc.huberlin.de/bitstream/handle/18452/13008/cclm.1986.24.1.49.pdf?sequence=1

MacDonald V (2009). Chemotherapy: Managing side effects and safe handling. The Canadian Veterinary Journal, 50(6): 665-668. Available at: https://www.ncbi.nlm.nih.gov/pmc/articles/PMC2684058/

Maisano R, Zavettieri M, Azzarello D, Raffaele M, Maisano M, Bottari M, and Nardi M (2011). Carboplatin and Gemcitabine Combination in Metastatic Triple-negative Anthracycline and Taxate- Pretreated Breast Cancer Patient: a Phase II study. Journal of Chemotherapy, 23: 40-43. Available at: https://www.tandfonline.com/doi/abs/10.1179/joc.2011.23.1.40

Marelli SP, Polli M, Frattini S, Cortellari M, Rizzi R, and Crepaldi P (2020). Genotypic and allelic frequencies of MDR1 gene in dogs in Italy. Veterinary Record Open, 7(1): 1-5. Available at: https://europepmc.org/article/med/32617164

Martinez-Ruzafa I, Dominguez PA, Dervisis NG, Sarbu L, Newman RG, Cadile CD, and Kitchell BE (2009). Tolerability of gemcitabine and carboplatin doublet therapy in cats with carcinomas. Journal of Veterinary Internal Medicine, 23(3): 570-577. DOI: https://www.doi.org/10.1111/j.1939-1676.2009.0279.x

McMahon M, Mathie T, Stingle N, Romansik E, Vail D, and London C (2011). Adjuvant Carboplatin and Gemcitabine Combination Chemotherapy Postamputation in canine Appendicular osterosarcoma. Journal of Veterinary Internal Medicine, 25: 511-517. DOI: https://www.doi.org/10.1111/j.1939-1676.2011.0697.x

McWhinney SR, Goldberg RM, and McLeod HL (2009). Platinum neurotoxicity pharmacogenetics. Molecular Cancer Therapeutics, 8: 10-16. Available at: https://mct.aacrjournals.org/content/molcanther/8/1/10.full.pdf

Mealey KL (2004). Therapeutic implications of the MDR-1 gene. Journal of Veterinary Pharmacology and Therapeutics, 27(5): 257-264. DOI: https://www.doi.org/10.1111/j.1365-2885.2004.00607.x

Mealey KL, Dassanayake S, and Burke NS (2017). Establishment of a cell line for assessing drugs as canine P-glycoprotein substrates: proof of principle. Journal of Veterinary Pharmacology and Therapeutics, 40(5): 545-551. DOI: https://www.doi.org/10.1111/jvp.12390

Medeiros VB (2017). Câncer da mama na cadela. Journal of Surgical and Clinical Research, 8: 118-129. DOI: https://www.doi.org/10.20398/jscr.v8i1.13039

Owen LN (1980). Mammary Glands. In: Owen LN (Editor), TNM Classification of tumours in Domestic Animals. 1st Ed. World Health Organization, Geneva, Switzerland, pp. 16-20.

Rasotto R, Berlato D, Goldschmidt MH and Zappulli V (2017). Prognostic significance of canine mammary tumor histologic subtypes: an observational cohort study of 229 cases. Veterinary Pathology, 54(4): 571-578. Available at: https://journals.sagepub.com/doi/10.1177/0300985817698208

Repetti CFS, Daleck CR (2007). Associação do methimazole e do ondansetron à quimioterapia com cisplatina em cães submetidos a quatro diferentes protocolos de fluidoterapia. Arquivo Brasileiro de Medicina Veterinária e Zootecnia, 59 (3): 665-672. Available at: https://www.scielo.br/scielo.php?script=sci_abstract\&pid=S0102-09352007000300018\&lng=en\&nrm=iso\&tlng=en

Rodaski S, and De Nardi (2008). Quimioterapia Antineoplásica em cães e gatos. 3rd Ed. MedVet, São Paulo, SP, Brasil, pp. 305.

Rodaski S, De Nardi AB, and Piekarz CH (2008). Quimioterapia Antineoplásica. In: Daleck CR, De Nardi AB, Rodaski S (Editors), Oncologia em cães e gatos. 1st Ed. Roca, São Paulo, SP, Brasil, pp. 161-177.

Saif M (2010). Pulmonary toxicity associated with gemcitabine. Journal of the Pancreas, 11(2): 189-190. Available at: http://www.serena.unina.it/index.php/jop/article/view/3859

Scheffé H (1999). The Analysis of Variance. 1st Ed. Wiley, New York, USA, p. 53.

Selmic LE, Burton JH, Thamm DH, Withrow SJ, and Lana SE (2014). Comparison of carboplatin and doxorubicin-based chemotherapy protofols in 470 dogs after amputation for treatment of appendicular osteosarcoma. Journal of Veterinary Internal Medicine, 28(2): 554-563. Available at: https://www.ncbi.nlm.nih.gov/pmc/articles/PMC4857984/

Silva AA, Carlotto J, and Rotta I (2018). Padronização da ordem de infusão de medicamentos antineoplásicos utilizados no tratamento dos cânceres de mama e colorretal. Einstein, 16(2): 1-9. Available at: https://www.scielo.br/pdf/eins/v16n2/pt_1679-4508-eins-16-02-eRW4074.pdf

Sorenmo KU, Worley DR, and Zappulli V (2020). Tumors of the Mammary Gland. In: Vail DM, Thamm DH, Liptak JM (Editors), Withrow \& MacEwen's Small Animal Clinical Oncology. 6th Ed. Saunders Elsevier, St. Louis, Missouri, USA, pp. 604-625.

Thamm DH, and Gustafson DL (2020). Drug dose and drug choice: Optimizing medical therapy for veterinary cancer. Veterinary and Comparative 
Thamm DH, and Vail DM (2007). Aftershocks of cancer chemotherapy: managing adverse effects. The American Animal Hospital Association, 43: 17. DOI: https://www.doi.org/10.5326/0430001

Toschi L, Finochiaro G, Bartolini S, Gioia V, and Cappuzzo F (2005). Role of gemcitabine in cancer therapy. Future Oncology, 1: 7-17. Available at: https://www.futuremedicine.com/doi/full/10.1517/14796694.1.1.7

Usami N, Yokoi K, Hasegawa Y, Taniguchi H, Shindo J, Yamamoto M, Suzuki R, Imaizumi K, Kondo M, and Shimokata K (2010). Central Japan Lung Study Group: phase II study of carboplatin and gemcitabine as adjuvant chemotherapy in patients with completely resected non-small cell lung cancer: a report from the Central Japan Lung Study Group. International Journal of Clinical Oncology, 15: 583-587. Available at: https://link.springer.com/article/10.1007/s10147-010-0118-x

Vail DM (2009). Supporting the Veterinary Cancer Patient on Chemotherapy: Neutropenia and Gastrointestinal Toxicity. Topics in Companion Animal Medicine, 24(3): 122-129. Available at: https://www.sciencedirect.com/science/article/abs/pii/S1938973609000154

Viana FAB (2007). Guia terapêutico veterinário. 2nd Ed. Cem, Lagoa Santa, MG, Brasil, pp. 463. Available at: https://www.magazineluiza.com.br/guia-terapeutico-veterinario-4-edicao-editora-cem/p/eja6c90hc4/li/ldvt/

Wagstaff AJ, Ward A, Benfield P, and Heel RC (1989). Carboplatin. A Preliminary Review of its Pharmacodynamic and Pharmacokinetic Properties and Therapeutic Efficacy in the Treatment of Cancer. Drugs, 37: 162-190. Available at: https://link.springer.com/article/10.2165/00003495198937020-00005

Wang S, Zhang H, Cheng L, Evans C, and Pan CX (2010). Analysis of the cytotoxic activity of carboplatin and gemcitabine combination. Anticancer Ressearch, 30(11): 4573-4578. Available at: https://www.ncbi.nlm.nih.gov/pmc/articles/PMC4562399/pdf/nihms720106.pdf 